\title{
Meningitis caused by Mycobacterium tuberculosis in a recent immigrant to Canada
}

\author{
Adam S. Komorowski MD, Carson K.L. Lo MD, Neal Irfan PharmD, Nishma Singhal MD
}

Cite as: CMAJ 2021 November 29;193:E1807-10. doi: 10.1503/cmaj.210740

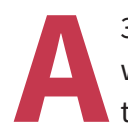

37-year-old woman presented to a community hospital with a 1-week history of severe frontal headache, subjective fever and episodic confusion. She denied having nausea, vomiting, seizures or rash. She had immigrated to Canada from Nigeria 8 months before presentation, with no travel since, and had no sick contacts or animal exposures.

On admission, the patient was febrile $\left(38.3^{\circ} \mathrm{C}\right)$, but vital signs were otherwise unremarkable. She had nuchal rigidity without focal neurologic deficits. Bloodwork showed a normal leukocyte count and an absolute lymphocyte count of 0.7 (normal 1.03.57) $\times 10^{9}$ cells/L. Test results for HIV were negative, as were blood smears for malaria parasites. Initial cerebrospinal fluid

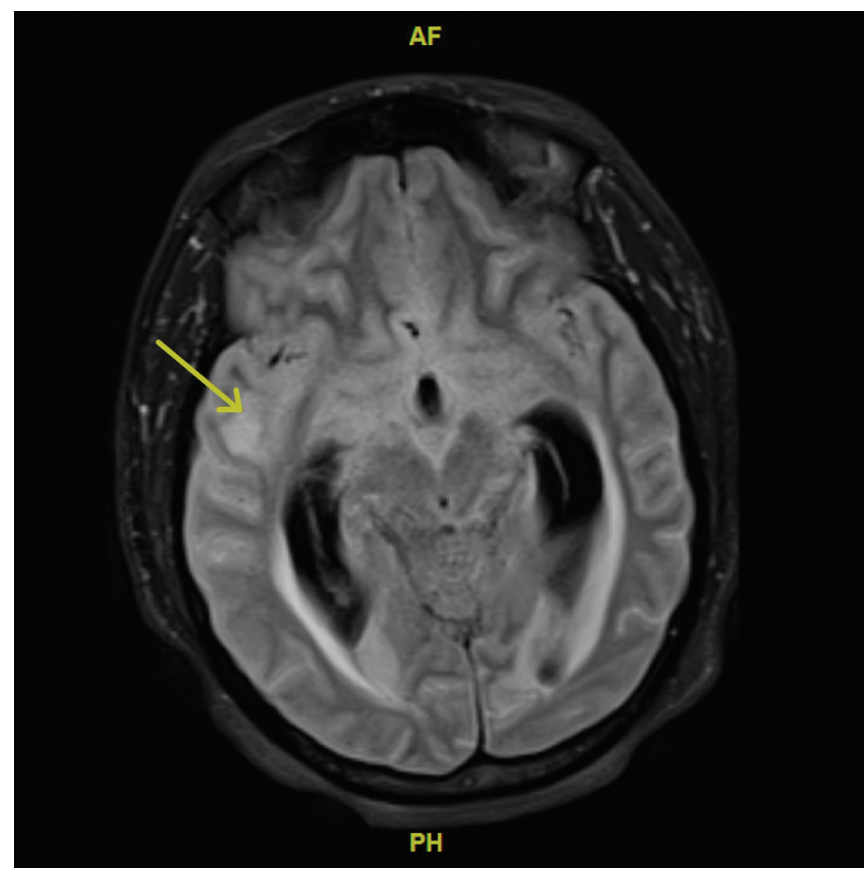

Figure 1: Magnetic resonance imaging (MRI) scan of the brain of a 37-year-old woman with tuberculous meningitis, obtained on day 5 of hospital admission. This axial fluid-attenuated inversion recovery (FLAIR) image at the level of the basal cisterns and temporal lobes shows high signal intensity throughout the convexity sulci and basal cisterns, compatible with meningitis. Both temporal horns of the lateral ventricles are dilated, with abnormal signal intensity surrounding them, compatible with hydrocephalus and transependymal passage of cerebrospinal fluid. The initial MRI report commented on possible parenchymal abnormality in the right temporal lobe (yellow arrow), which was thought to be compatible with encephalitis. Upon review of this image, however, the MRI findings are most compatible with meningitis.

\section{Key points}

- In Canada, tuberculous meningitis is an uncommon presentation of Mycobacterium tuberculosis infection, occurring most frequently in individuals who arrived to Canada within the previous 5 years.

- In patients with risk factors for tuberculosis (TB), a presumptive diagnosis of TB meningitis should be considered if clinical presentation and findings from cerebrospinal fluid are consistent; early empiric therapy should be initiated.

- Clinicians should monitor patients closely for adverse reactions to antituberculous medications, such as transaminitis and acute kidney injury, and stop potentially hepatotoxic drugs, where possible.

- Patients with chronic hydrocephalus as a complication of their meningitis may require a protracted course of corticosteroids, in addition to extraventricular drainage.

(CSF) studies showed a leukocyte count of 96 (normal $0-5) \times 10^{6}$ cells/L (59\% lymphocytes, 36\% neutrophils and $2 \%$ monocytes), glucose of 1.9 (normal 2.2-3.9) mmol/L and protein of 1.20 (normal < 0.45 ) g/L. At admission, her aspartate aminotransferase was 13 (normal 10-40) U/L, and her alanine aminotransferase was 18 (normal 5-42) U/L. Results from CSF bacterial and fungal cultures, polymerase chain reaction for herpes simplex virus and enterovirus, cryptococcal antigen lateral flow assay and autoimmune encephalitis work-up were negative. Mycobacterial culture of the CSF was not requested.

Concern for meningoencephalitis prompted a noncontrast computed tomography (CT) scan of the patient's head, which showed a small area of subcortical hypodensity in the right temporal lobe, suspicious for encephalitis. Her clinicians started empirical treatment with ceftriaxone, vancomycin and acyclovir. On day 5 , she became nonverbal and confused, and developed a new left lateral rectus palsy and an ipsilateral, nonreactive pupil measuring $6 \mathrm{~mm}$. Brain magnetic resonance imaging showed high signal intensity throughout the convexity sulci and basal cisterns, compatible with meningitis (Figure 1). A CT scan of the chest, abdomen and pelvis showed nonspecific abdominal lymphadenopathy. She developed Cushing reflex and was intubated for transfer to our tertiary hospital for neurosurgical intervention. Neurosurgeons inserted an external ventricular drain to relieve hydrocephalus, and escalated antimicrobial therapy to meropenem, vancomycin and acyclovir. 


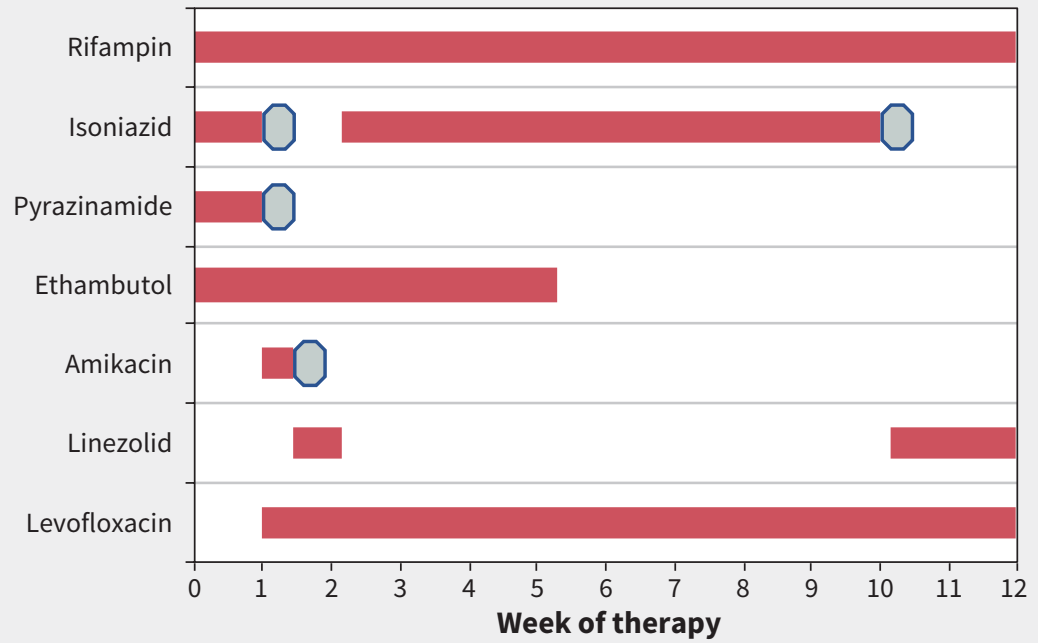

Figure 2: Schematic representation of changes to the patient's treatment for tuberculous meningitis in the first 3 months after diagnosis, with adverse events from antimicrobials, represented by octagons. Asymptomatic elevation of aminotransferases greater than 5 times the upper limit of normal led to stopping isoniazid and pyrazinamide. We added amikacin, but stopped it after 3 days because of the patient's acute kidney injury. We used linezolid as a bridging therapy until the patient's aminotransferase elevation had resolved; we then reintroduced isoniazid. We eventually stopped ethambutol because the patient's Mycobacterium tuberculosis was found to be sensitive to rifampin and isoniazid. At week 10 of therapy, the patient developed hepatitis, requiring us to stop isoniazid permanently and to reintroduce linezolid.

We consulted the infectious diseases team, who considered Mycobacterium tuberculosis meningitis to be the primary differential diagnosis given the patient's risk factors, progressive presentation despite treatment with antimicrobial agents, CSF biochemical findings and abdominal lymphadenopathy. We stopped antimicrobial therapy, performed another lumbar puncture to obtain CSF for mycobacterial culture and GeneXpert MTB/RIF nucleic acid amplification testing (NAAT; Cepheid), and started empirical treatment for tuberculous (TB) meningitis with intravenous (IV) dexamethasone $0.4 \mathrm{mg} / \mathrm{kg} / \mathrm{d}$ divided every 4 hours, IV rifampin $10 \mathrm{mg} / \mathrm{kg} / \mathrm{d}$, oral isoniazid $5 \mathrm{mg} / \mathrm{kg} / \mathrm{d}$, oral pyrazinamide $25 \mathrm{mg} / \mathrm{kg} / \mathrm{d}$ and oral ethambutol $15 \mathrm{mg} / \mathrm{kg} / \mathrm{d}$. Acid-fast bacilli were not seen on CSF staining, and NAAT was negative for M. tuberculosis complex. Our intensivists performed bronchoscopy, from which NAAT, staining and culture were ultimately negative.

Beginning on day 18 , adverse events necessitated a series of changes to the patient's treatment regimen (Figure 2). Her levels of aspartate aminotransferase $(141 \mathrm{U} / \mathrm{L})$ and alanine aminotransferase $(227 \mathrm{U} / \mathrm{L})$ were more than 5 times the upper limit of normal, which prompted us to stop pyrazinamide and isoniazid. We substituted with IV amikacin $15 \mathrm{mg} / \mathrm{kg} / \mathrm{d}$ and IV levofloxacin $10 \mathrm{mg} / \mathrm{kg} / \mathrm{d}$. Amikacin caused acute kidney injury, with a doubling of serum creatinine over 4 days, so we switched to oral linezolid $600 \mathrm{mg}$ twice daily. Once transaminase levels returned to normal, we stopped linezolid and reintroduced isoniazid. As the patient later developed a transaminitis that we attributed to isoniazid, we permanently stopped isoniazid and reintroduced linezolid.

M. tuberculosis was detected in liquid culture of CSF on day 37. The isolate was negative for mutations causing resistance to rifampin and isoniazid, and the laboratory later confirmed susceptibility to first-line (i.e., isoniazid, rifampin, pyrazinamide, ethambutol) and second-line (i.e., ethionamide, linezolid, moxifloxacin) agents.

Our initial attempts to taper the patient off of dexamethasone according to routine schedules were unsuccessful because of persistent hydrocephalus; she continued dexamethasone treatment during her hospitalizations and was switched to hydrocortisone upon discharge to allow for a slow taper of steroids over 5 months (Figure 3). The patient's external ventricular drain was weaned and eventually removed on day 31 . On day 35 , she was extubated; shortly thereafter, however, neurosurgeons reinserted an external ventricular drain for worsening hydrocephalus and ultimately exchanged it for a programmable ventriculoperitoneal shunt (VPS). This was later replaced with a mediumpressure valve VPS after a short period of worsening hydrocephalus and generalized myoclonus concerning for seizures. The patient was eventually discharged home on day 148 from the community hospital.

The patient remained stable at 1-year follow-up, and her antituberculous medications were stopped. Though she recovered functional independence and regained verbal communication skills, she continues to suffer from disease sequelae and treatment-related complications, particularly cerebral salt wasting syndrome and bilateral optic nerve damage, and has been unable to return to work.

\section{Discussion}

Tuberculosis remains a leading cause of death globally, with 1.4 million deaths in 2019. ${ }^{1}$ In Canada, most reported cases of TB are among foreign-born individuals, with more than $40 \%$ occurring within the first 5 years of arrival. ${ }^{1}$ Tuberculosis of the central ner- 


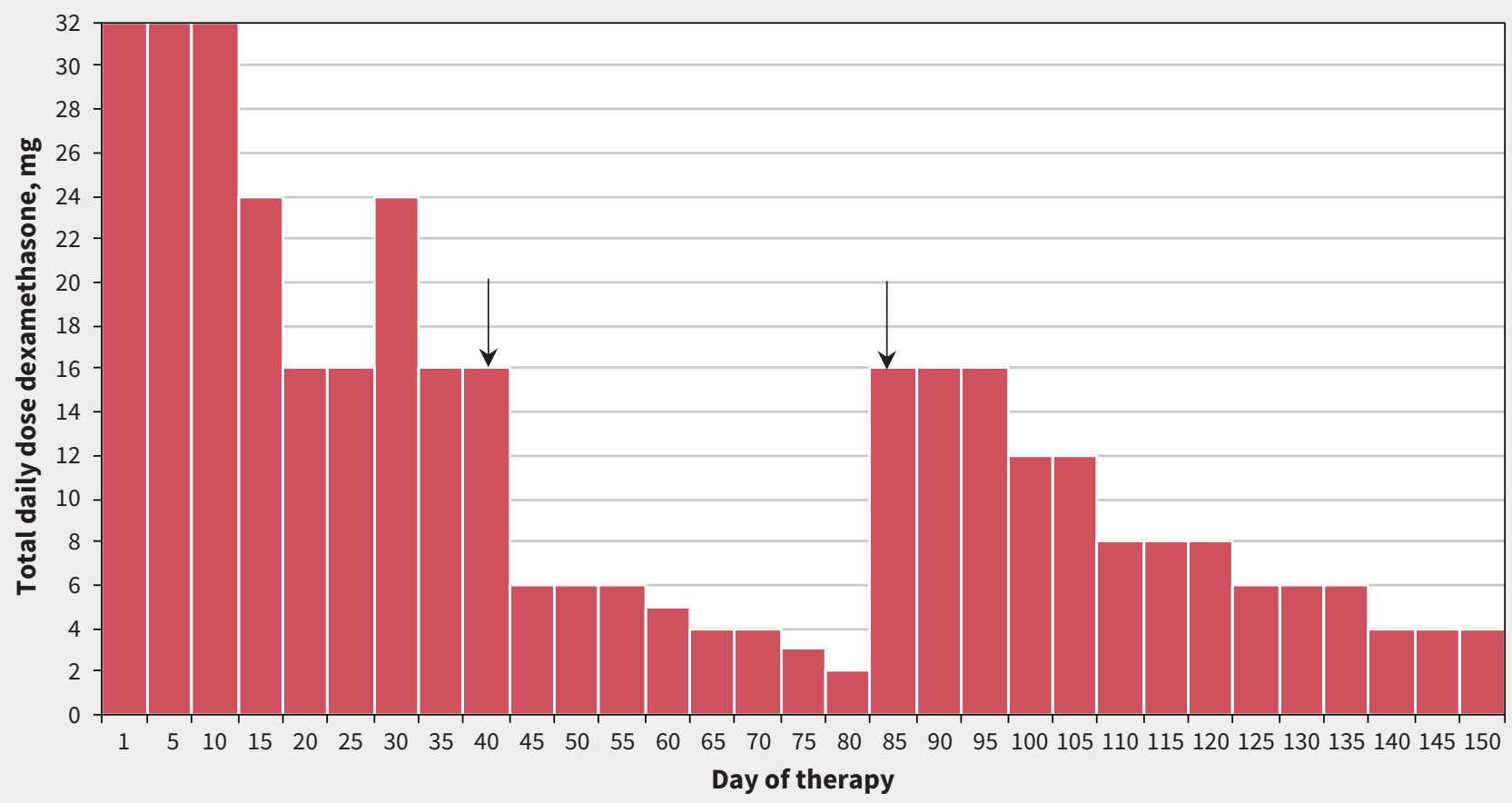

Figure 3: Total daily dose of dexamethasone during the patient's hospitalization for tuberculous meningitis. From day 1 to day 40 , the patient was cared for at our tertiary care centre; the arrow at day 40 indicates her transfer back to another hospital, where she stayed until day 85 . The arrow at day 85 indicates her readmission to our tertiary care centre for worsening hydrocephalus. She was transferred back to the other hospital on day 87 , where she remained an inpatient until discharge on day 148. The patient was prescribed hydrocortisone as an outpatient, which was slowly tapered over 5 months.

vous system accounts for $1 \%-2 \%$ of all TB cases, causing a spectrum of disease that includes brain abscess, cerebritis, meningitis, myelitis and tuberculomas. Tuberculous meningitis is the most common central nervous system manifestation, with substantial morbidity $(25 \%)$ and mortality $(15 \%-40 \%)$ rates, even with appropriate treatment. ${ }^{2}$ Independent risk factors for death among inpatients with TB meningitis include HIV infection, age older than 40 years, CSF culture positive for TB and disease severity. ${ }^{3}$

Tuberculous meningitis typically presents subacutely, with fever, headache, malaise and personality changes, which can progress to cranial nerve palsies or other focal neurologic deficits, seizures, coma and death. Lumbar puncture is vital, as CSF findings can be suggestive of a diagnosis of TB meningitis and prompt the decision to start empiric therapy. Typical CSF findings include an elevated opening pressure, marked lymphocytic pleocytosis, elevated protein caused by obstructed CSF flow, and low glucose.

Microbiologic testing is confirmatory in the diagnosis of TB meningitis, but it has limitations. Samples of CSF should be sent for acidfast bacillus staining and microscopy, NAAT and culture. However, the mycobacterial burden in CSF is generally lower in patients with TB meningitis than with pulmonary TB, which affects the yield from microbiologic testing. The sensitivity of staining for acid-fast bacillus and microscopy (20\%-80\%) is lower in patients with extrapulmonary TB and in patients with HIV infection; thus, a negative result from acid-fast bacillus staining of CSF should not lead clinicians to rule out TB meningitis. ${ }^{1}$ However, the sensitivity of staining for acid-fast bacillus in TB meningitis can be increased by performing daily lumbar punctures for 3 days, submitting the last tube collected and collecting a large sample volume $(10-15 \mathrm{~mL}) .{ }^{1}$ Similarly, NAAT testing, which has the advantage of providing a presumptive diagnosis if positive, with a turnaround time of less than 24 hours, has a lower sensitivity in CSF than sputum. ${ }^{2,4}$ Concomitant pulmonary TB is found in $22 \%-24 \%$ of patients with TB meningitis, and sputum examination offers a less invasive diagnostic modality; therefore, patients with presumed TB meningitis should be investigated for pulmonary TB. ${ }^{1}$ In our case, work-up for pulmonary TB was negative.

Mycobacterial cultures can take up to 7 weeks and a negative culture cannot exclude TB meningitis in patients without an alternative diagnosis who have improved on antituberculous therapy. Therefore, if clinical presentation and CSF findings are consistent, a presumptive diagnosis of TB meningitis should be considered for patients with risk factors for TB. It is imperative that early empiric therapy is initiated, as time to initiation of appropriate antituberculous therapy before coma onset is the strongest predictor of survival. ${ }^{5}$

Current guidelines recommend 2 months of intensive phase treatment with rifampin, isoniazid, pyrazinamide and ethambutol for drug-susceptible TB, followed by continuation phase treatment with rifampin and isoniazid for an additional 10 months. ${ }^{1,4,6}$ No difference in clinical outcome or relapse has been found with a 6-month treatment duration. ${ }^{7}$ Rifampin and ethambutol remain cornerstones of intensive phase therapy for TB meningitis because small trials have failed to show an improvement in mortality rates when better CSF-penetrating agents are added. ${ }^{8}$ 
As shown with our patient, antituberculous therapy requires careful monitoring for adverse events. When encountered, identification of the most likely causative agent, a decision about its temporary cessation or permanent discontinuation, and choice of an acceptable alternative is important. Clinicians should consider second-line agents with acceptable CSF pharmacokinetic parameters, such as levofloxacin, linezolid, moxifloxacin, cycloserine or ethionamide. These parameters led us to choose a twice daily linezolid dosage during the patient's critical illness while susceptibility testing results were unavailable, as opposed to the once daily dosage typically used in trials of TB meningitis. One study ${ }^{9}$ showed improvement in CSF biochemical parameters and Glasgow Coma Scale with the regimen we used.

Potentially hepatotoxic drugs should be stopped when serious transaminitis occurs. ${ }^{10}$ Guidelines recommend stopping pyrazinamide, isoniazid and rifampin, and substituting with either a fluoroquinolone and an injectable agent (e.g., aminoglycosides, capreomycin), or a fluoroquinolone and an oral second-line agent (e.g., linezolid). ${ }^{1}$ We withheld the 2 most likely causes of transaminitis, isoniazid and pyrazinamide, but continued rifampin to avoid stopping most first-line agents during our patient's critical illness.

Successful treatment of TB meningitis requires a multimodal approach to manage increased intracranial pressure, including corticosteroids, which have been shown to reduce risk of death. ${ }^{5,11}$ The optimal dose and duration of corticosteroids is unclear, with guidelines suggesting a 6-8 week taper., ${ }^{1,4,6}$ Our case highlights the limitation of these recommendations and the importance of tailoring an individual's therapy, as multiple attempts to wean the patient's corticosteroids failed; she ultimately required a 48-week taper.

Management of associated hydrocephalus, especially if noncommunicating, ${ }^{12}$ may require urgent neurosurgical intervention such as an external ventricular drain for short-term control and - as in our patient - a VPS for definitive management. ${ }^{11}$ Optic nerve complications are responsible for most vision loss associated with TB meningitis and can have multiple causes, but were attributed to chronic hydrocephalus in our patient given the time of onset..$^{13}$ Hyponatremia can be caused by cerebral salt wasting syndrome or syndrome of inappropriate antidiuretic hormone secretion, which need to be distinguished because of their different treatment approaches. ${ }^{12}$ Our patient's hyponatremia was attributed to cerebral salt wasting syndrome, and corrected with oral supplementation of sodium chloride.

Our case shows the diagnostic and therapeutic challenges of managing TB meningitis. Early recognition and institution of appropriate therapy, with continued monitoring throughout the disease course, maximizes the probability of functional recovery.

\section{References}

1. Canadian tuberculosis standards 7th edition: 2014. Ottawa: Public Health Agency of Canada; 2014. Available: https://www.canada.ca/en/public-health/ services/infectious-diseases/canadian-tuberculosis-standards-7th-edition.html (accessed 2021 Apr. 17).

2. Thwaites GE, Tran TH. Tuberculous meningitis: many questions, too few answers. Lancet Neurol 2005;4:160-70.

3. Soria J, Metcalf T, Mori N, et al. Mortality in hospitalized patients with tuberculous meningitis. BMC Infect Dis 2019;19:9.

4. Guidelines for treatment of drug-susceptible tuberculosis and patient care, 2017 update. Geneva: World Health Organization; 2017. Available: https:// apps.who.int/iris/handle/10665/255052 (accessed 2021 Apr. 17).
5. Prasad K, Singh MB, Ryan H. Corticosteroids for managing tuberculous meningitis. Cochrane Database Syst Rev 2016;(4):CD002244.

6. Nahid P, Dorman SE, Alipanah N, et al. Official American Thoracic Society/ Centers for Disease Control and Prevention/Infectious Diseases Society of America Clinical practice guidelines: treatment of drug-susceptible tuberculosis. Clin Infect Dis 2016;63:e147-95.

7. Jullien S, Ryan H, Modi M, et al. Six months therapy for tuberculous meningitis. Cochrane Database Syst Rev 2016;(9):CD012091.

8. Rizvi I, Malhotra HS, Garg RK, et al. Fluoroquinolones in the management of tuberculous meningitis: systematic review and meta-analysis. J Infect 2018;77:261-75.

9. Sun F, Ruan Q, Wang J, et al. Linezolid manifests a rapid and dramatic therapeutic effect for patients with life-threatening tuberculous meningitis. Antimicrob Agents Chemother 2014;58:6297-301.

10. Saukkonen JJ, Cohn DL, Jasmer RM, et al. An official ATS statement: hepatotoxicity of antituberculosis therapy. Am J Respir Crit Care Med 2006;174:935-52.

11. Davis A, Meintjes G, Wilkinson RJ. Treatment of tuberculous meningitis and its complications in adults. Curr Treat Options Neurol 2018;20:5. doi: 10.1007/ s11940-018-0490-9.

12. Donovan J, Figaji A, Imran D, et al. The neurocritical care of tuberculous meningitis. Lancet Neurol 2019;18:771-83.

13. Garg RK, Malhotra HS, Kumar N, et al. Vision loss in tuberculous meningitis. J Neurol Sci 2017;375:27-34.

\section{Competing interests: None declared.}

This article has been peer reviewed.

The authors have obtained patient consent.

Affiliations: Division of Medical Microbiology (Komorowski), Department of Pathology and Molecular Medicine; Clinician-Investigator Program (Komorowski); Division of Infectious Diseases (Lo, Irfan, Singhal), Department of Medicine, McMaster University, Hamilton, Ont.

Contributors: Adam Komorowski was responsible for conceptualization, and Adam Komorowski and Carson Lo contributed to the methodology; Neal Irfan and Nishma Singhal were responsible for supervision. Adam Komorowski and Carson Lo wrote the original draft. All authors revised it critically for important intellectual content, gave final approval of the version to be published and agreed to be accountable for all aspects of the work.

Content licence: This is an Open Access article distributed in accordance with the terms of the Creative Commons Attribution (CC BY-NCND 4.0) licence, which permits use, distribution and reproduction in any medium, provided that the original publication is properly cited, the use is noncommercial (i.e., research or educational use), and no modifications or adaptations are made. See: https://creativecommons.org/ licenses/by-nc-nd/4.0/

Acknowledgements: The authors thank the patient for their permission to publish this case report. They also wish to thank Drs. Sarah Brode, Ian Kitai, Julianne Kus and Anjali Shroff for their invaluable expertise and initial advice related to this patient's care, and Dr. Ryan Rebello for his assistance with the Figure 1 caption.

Correspondence to: Adam Komorowski, adam.komorowski@medportal.ca

The section Cases presents brief case reports that convey clear, practical lessons. Preference is given to common presentations of important rare conditions, and important unusual presentations of common problems. Articles start with a case presentation (500 words maximum), and a discussion of the underlying condition follows (1000 words maximum). Visual elements (e.g., tables of the differential diagnosis, clinical features or diagnostic approach) are encouraged. Consent from patients for publication of their story is a necessity. See information for authors at www.cmaj.ca. 